\title{
Clima e suporte organizacional: avaliação e relação entre as temáticas
}

\author{
Climate and Organizational Support: Evaluation and \\ Relationship between the Thematic Areas
}

Clima y Soporte Organizacional: Evaluación y relación entre las temáticas

Hugo Ferrari Cardoso ${ }^{1} \underline{\mathrm{ORCID}}$, Julia Basile Forlini ${ }^{2 \text { Lattes }}$, Jhenifer Prescilla Dias ${ }^{3 凶} \underline{\mathrm{ORCID}}$

Universidade Estadual Paulista (UNESP)

Brasil

Fecha correspondencia:

Recibido: enero 25 de 2018.

Aceptado: enero 22 de 2019.

Forma de citar:

Ferrari Cardoso, H., Basile Forlini, J., \& Prescilla Dias, J. (2019). Clima e suporte organizacional: avaliação e relação entre as temáticas. Rev.CES Psico, 12(2), 65-82.

\section{Open access}

(c) Copyright

Licencia creative commons

Etica de publicaciones

Revisión por pares

Gestión por Open Journal System

DOI: http://dx.doi.org/10.21615/

cesp. 12.2 .6

ISSN: 2011-3080

\section{Sobre los autores:}

1. Pós-Doutor em Psicología. Docente de Psicología de la Universidade Estadual Paulista (UNESP), Brasil.

Comparte

\section{F日G.898 $\mathbb{R}^{0}$ 因}

\section{Resumo}

É cada vez mais comum o discurso de que as organizações visam proporcionar melhores condições de trabalho a seus funcionários, o que pode ser entendido como uma busca crescente pelo oferecimento de suporte e melhorias quanto à percepção de adequado clima de trabalho. Nesse sentido, a presente pesquisa investigou a percepção de clima e suporte organizacionais em uma amostra de 300 trabalhadores, analisou-se também possíveis diferenças de médias de respostas em relação a variáveis sociodemográficas com base nas pontuações obtidas pelos trabalhadores nos instrumentos aplicados: Escala de Clima Organizacional (CLIMOR) e à Escala de Suporte Laboral (ESUL), bem como verificou-se as correlações entre as temáticas clima e suporte organizacionais. Dos resultados, os participantes apresentaram pontuações classificadas com Média-Baixa, Média-Alta e Alta (ESUL) e Média-Baixa (CLIMOR), houve diferenças significativas em relação a variável tipo de organização e as associações entre os instrumentos foram positivas, indicando que maior percepção de um adequado clima organizacional tende a estar associada a maiores indicadores de suporte organizacional. Esses e outros resultados são discutidos com base na literatura.

Palavras chave: Clima Organizacional, Suporte Organizacional, Psicologia Organizacional e do Trabalho, Comportamento Organizacional, Gestão de Pessoas, Bem-estar no Trabalho.

\section{Abstract}

It is increasingly common the statement about organizations are aimed to provide better working conditions for their employees, it can be understood as a growing search to provide support and improve the perception of a suitable work climate. In this sense, this the present study investigated the perception of climate and organizational support in a sample of 300 workers; it was also analyzed possible differences in the average of responses in relation to sociodemographic variables based on the scores 
Pág 66

2. Psicóloga Universidade Estadual Paulista (UNESP), Brasil.

3. Maestranda em Psicología. Psicóloga. Psicóloga Escolar no Colégio ADV (Unidade II - Jaú) e Psicóloga responsável pelo Serviço Universitário de Apoio Psicopedagógico ao aluno (SUAPP) na Universidade do Oeste Paulista (UNOESTE campus Jaú). obtained by the workers in the applied instruments: Climate Scale (CLIMOR) and the Labor Support Scale (ESUL); as well as correlations between the areas of organizational climate and support. The participants' scores classified as Medium-Low, Medium-High and High (ESUL) and Medium-Low (CLIMOR), there were significant differences regarding the type of organization variable, and the associations between the instruments were positive, indicating that greater perception of suitable work climate tends to be associated with higher indicators of organizational support. These and other results are discussed on the basis of literature.

Keywords: Organizational Climate, Organizational Support, Organizational and Work Psychology, Organizational Behavior, People Management, Well-being at Work.

\section{Resumen:}

Es cada vez más común el discurso respecto a que las organizaciones apuntan a proporcionar mejores condiciones de trabajo a sus funcionarios, lo que puede entenderse como una búsqueda creciente por ofrecer soporte y mejorar la percepción de un adecuado clima de trabajo. En este sentido, el presente estudio investigó la percepción del clima y soporte organizacional en una muestra de 300 trabajadores, se analizaron también posibles diferencias de promedios de respuestas en relación a variables sociodemográficas con base en las puntuaciones obtenidas por los trabajadores en los instrumentos aplicados: Escala de Clima (CLIMOR) y Escala de Soporte Laboral (ESUL), y además, se verificaron las correlaciones entre las temáticas clima y soporte organizacional.). Los participantes presentaron puntuaciones clasificadas con Media-Baja, Media-Alta y Alta (ESUL) y Media-Baja (CLIMOR), se encontraron diferencias significativas en relación a la variable tipo de organización, y las asociaciones entre los instrumentos fueron positivas, indicando que una mayor percepción de un adecuado clima organizacional tiende a estar asociada a mayores indicadores de soporte organizacional. Estos y otros resultados se discuten con base en la literatura.

Palabras clave: Clima Organizacional, Soporte Organizacional, Psicología Organizacional y del Trabajo, Comportamiento Organizacional, Gestión de Personal, Bienestar en el Trabajo.

\section{Introdução}

Com a globalização e o advento das inovações tecnológicas, o cenário do mundo do trabalho cada vez mais vem se apresentando em crescente competividade, sendo o funcionário cobrado pela eficiência e rapidez no desenvolvimento de suas atividades, pela constante qualificação profissional e pelo comprometimento com a organização em que trabalha (Simões, Alves, \& Silva, 2016). Ao longo de sua vida, o ser humano tende a dedicar grande parte do seu tempo ao trabalho, o qual acaba por tornar-se uma área quase central em sua vida. Essa centralidade traz consequências que podem ser consideradas paradoxais (Azevedo \& Tonell, 2014), pois à medida que o trabalho pode proporcionar muitas realizações dentro dos contextos organizacionais, também pode representar uma fonte de adoecimento psíquico.

Investigações acerca das mudanças no âmbito do trabalho, e que repercutem na vida dos indivíduos, ganham, gradativamente, mais destaque no meio profissional e acadêmico. Há indícios de que um estado prolongado de estresse interfere no bem-estar psicológico e na qualidade de vida das pessoas (Amazue \& Onyishi, 2016. Shoss, Eisenberger, Restubog, \& Zagenczyk, 2013, Viitala, Tanskanen, \& Säntti, 2015). 
Quando tal clima é percebido como inadequado (negativo) pode, ao longo do tempo, levar o trabalhador à insatisfação e até mesmo a apresentar comportamentos de fuga do trabalho, como absenteísmo, afastamento por questões de saúde ou mesmo pedidos de demissão.
Com base no exposto, percebe-se que tanto em pesquisas científicas, como na prática profissional de psicólogos inseridos nas organizações de trabalho, há o aumento da valorização de conceitos, tais como saúde do trabalhador, motivação, clima organizacional, suporte laboral, dentre outros (Zanelli \& Bastos, 2004). De forma específica, a presente introdução abordará o clima organizacional e suporte laboral, temáticas essas norteadoras deste estudo. Cabe destacar que no presente estudo serão utilizados suporte laboral e suporte organizacional como sinônimos, uma vez que a literatura científica da área não faz distinções teóricas entre tais terminologias.

No que diz respeito ao clima organizacional, de acordo com Shanker, Bhanugopan, Heijden e Farrell (2017), o mesmo se refere às formas como os trabalhadores percebem o contexto onde trabalham. Tais percepções podem ser influenciadas por fatores externos e internos à organização, não se restringindo a variáveis como motivação ou satisfação do funcionário, mas considera-se, também, o clima como eventos, práticas e processos da organização, ou seja, são contempladas diversas dimensões do ambiente.

Com base em Chermack, Coons, Nimon, Bradley e Glick (2015) e Nakata, Veloso, Fischer e Dutra (2009), pode-se afirmar que o clima organizacional é formado pelos sentimentos, positivos ou negativos, que os trabalhadores compartilham em relação à organização e que refletem, e também podem ser a causa, de determinados comportamentos no contexto de trabalho. Quando tal clima é percebido como inadequado (negativo) pode, ao longo do tempo, levar o trabalhador à insatisfação e até mesmo a apresentar comportamentos de fuga do trabalho, como absenteísmo, afastamento por questões de saúde ou mesmo pedidos de demissão.

É válido ressaltar que o clima organizacional reflete um estado momentâneo da organização, que pode alterar-se ante alguma mudança no ambiente ou no contexto e, portanto, pode-se dizer que as pesquisas de clima organizacional conseguem apurar as percepções que as pessoas têm sobre uma realidade num determinado momento (Menezes \& Gomes, 2010, Rueda \& Santos, 2011, Shmidt \& Frazier, 2016. Wallace et al., 2016). A análise do clima organizacional pode ser uma importante ferramenta utilizada pela área de Gestão de Pessoas (GP) da empresa na busca pela melhoria, por meio de ações planejadas, no que tange às interações entre trabalhadores, bem como das atividades laborais. Essas ações de GP podem fazer com que os funcionários percebam a organização como um local adequado de trabalho, podendo acarretar um aumento da percepção do suporte organizacional (Rueda et al., 2014).

Quanto a esse último conceito, suporte organizacional, o mesmo está relacionado às expectativas e crenças que os funcionários possuem sobre a retribuição e o reconhecimento que a organização the atribui. Diz respeito ao quanto os funcionários sentem-se valorizados, ou seja, têm a sensação de que a organização de trabalho promove ações relacionadas ao bem-estar dos mesmos (Eisenberger, Huntington, Hutngton, \& Sowa, 1986). A percepção do suporte laboral tende a fazer com que os empregados se percebam valorizados, pelos superiores e os próprios colegas de trabalho, e que fazem parte de um compromisso mútuo, de uma rede social dentro da organização. Essa rede social de apoio pode ser definida como o conjunto de pessoas com as quais um indivíduo se relaciona, se identifica socialmente e elege como referências para sua vida (Bowling, 1997, Culié, Khapova, \& Arthur, 2014, Ribeiro, 1999. Wikhamn \& Hall, 2014). 
Pág 68

Pesquisadores que estudam o suporte organizacional tendem a concordarem que o mesmo, se percebido de forma positiva, geralmente se associa à redução do absenteísmo e patologias laborais.
Nesse sentido, o conceito de suporte organizacional encontra-se alicerçado em duas vertentes teóricas, quais sejam, a teoria das trocas sociais e a teoria da norma da reciprocidade (Wayne et al. 2002, Aselage \& Eisenberger, 2003). A primeira concebe que as trocas sociais acontecem unicamente com base na confiança e na boa fé entre as partes (no caso, entre trabalhadores e organização), envolvendo a oferta de favores, que acabam por criar obrigações morais futuras (Wayne et al, 2002, Gomide Jr., Guimarães, \& Damásio, 2004). Já na segunda, menciona-se que as relações sociais são regidas por um princípio moral, aceito universalmente, o qual define a obrigação de retribuir favores recebidos. Assim é esperado que se deve ajudar a quem nos ajuda e não se deve prejudicar quem nos beneficia. Pesquisadores que estudam o suporte organizacional tendem a concordarem que o mesmo, se percebido de forma positiva, geralmente se associa à redução do absenteísmo e patologias laborais (Eisenberger, Fasolo, \& Davis-la Mastro, 1990, Eisenberger et al., 1986, Eisenberger, Stinglhamber, Vandenberghe, Sucharski, \& Rhoades, 2002, Gillet, Huart, Colombat, \& Fouquereau, 2013, Kurtessis et al., 2017, Sears, Zhang, \& Han, 2016, Wikhamn \& Hall, 2014).

Como visto anteriormente, é cada vez mais comum o discurso de que as organizações visam proporcionar melhores condições de trabalho a seus funcionários, o que pode ser entendido como uma busca crescente pelo oferecimento de suporte e melhorias quanto à percepção de adequado clima de trabalho. Nesse sentido, a presente pesquisa investigou a percepção de clima e suporte organizacionais em uma amostra de trabalhadores, analisou-se também possíveis diferenças de médias de respostas em relação a variáveis sociodemográficas (sexo do participante, idade, tipo de organização e ramo de atividade da organização) com base nas pontuações obtidas pelos trabalhadores nos instrumentos aplicados, bem como verificou-se as correlações entre as temáticas clima e suporte organizacionais.

\section{Método}

\section{Participantes}

A amostra foi comportas por 300 trabalhadores com idades entre 19 e 66 anos $(M=33,64, D P=11,14)$, sendo $172(57,3 \%)$ do sexo feminino e $128(42,7 \%)$ do sexo masculino. Quanto às informações sociodemográficas, em relação ao estado civil dos participantes a maioria era solteira, $(n=156,52 \%)$, seguido de casado ( $n=115$, $38,3 \%)$, separado $(n=22,7,3 \%)$, amasiado $(n=6,2 \%)$ e viúvo $(n=1,0,3 \%)$. No que se refere ao nível instrucional dos trabalhadores, a maior parte possuía ensino superior completo $(n=208,69,3 \%)$, seguido por trabalhadores com ensino superior incompleto $(n=49,16,3 \%)$, ensino médio completo $(n=33,11 \%)$, ensino fundamental completo $(n=5,1,7 \%)$, fundamental incompleto $(n=3,1 \%)$ e ensino médio incompleto $(n=2,0,7 \%)$.

Das informações laborais, a maior parte, 196 (65,3\%), era composta por trabalhadores de organizações do tipo privadas, seguido de pessoas que atuavam em organizações públicas $(n=85,28,3 \%)$ e terceiro setor $(n=18,6 \%)$. Em relação ao ramo de atuação das organizações, a maioria $(n=200,66,7 \%)$ era do ramo de prestação de serviços, e em menor frequência havia trabalhadores do ramo industrial $(n=57,19 \%)$ e comercial ( $n=43,14,4 \%)$. Também se investigou se os trabalhadores apresentavam mais de um emprego, tendo como resultados que a maioria $(n=239,79,7 \%)$ não possuía outro emprego e 61 (20,3\%) tinham mais de um emprego.

Acerca da classificação das organizações utilizou-se os critérios estabelecidos pelo Serviço Brasileiro de Apoio às Micro e Pequenas Empresas (SEBRAE, 2013), o qual 
estabelece que microempresas são as que possuem até 20 trabalhadores, as pequenas empresas são constituídas de 21 a 99 funcionários, médias empresas as que possuem de 100 a 499 empregados e as grandes empresas as que possuem acima de 500 trabalhadores. Nessa mesma direção, fizeram parte da amostra 74 (24,7\%) trabalhadores de microempresas, 75 (25\%) de pequenas empresas, 74 (24,7\%) de médias empresas e $77(25,7 \%)$ de grandes empresas.

\section{Instrumentos}

Escala de Suporte Laboral - ESUL (Baptista, Cardoso, Santos, \& Noronha, 2016) A ESUL avalia a percepção dos trabalhadores em relação ao suporte que recebe da organização em que trabalha. 0 instrumento possui 21 itens, agrupados em dois fatores, respondidos por meio de uma escala do tipo Likert de quatro pontos. 0 primeiro fator (Interação) se relaciona ao processo de vinculação do trabalhador com os demais colegas da organização, com a chefia e a organização, bem como qualidade no processo de circulação de informações na organização. Já o segundo fator (Benefícios) liga-se ao suporte de ordem material (suporte instrumental) recebido pela organização.

0 instrumento possui diversos estudos que atestam suas qualidades psicométricas e é reconhecido pelo Conselho Federal de Psicologia (CFP), por meio do Sistema de Avaliação de Testes Psicológicos (SATEPSI), como um teste psicológico. De acordo com o manual do instrumento (Baptista et al., 2016), para a sua correção deve-se levar em consideração o ramo de atuação da organização em que o indivíduo trabalha, ou seja, comércio, indústria e prestação de serviços. Com base nos escores obtidos pelos trabalhadores, o teste apresenta quatro possíveis classificações (Baixa, Médio-baixa, Médio-alta, Alta), de forma que quanto maior a pontuação, maior a percepção de suporte organizacional.

Escala de Clima Organizacional - CLIMOR (Rueda \& Santos, 2011)

A CLIMOR avalia a percepção do indivíduo em relação ao contexto de trabalho. A escala possui 32 itens, distribuídos em cinco fatores (Comunicação, integração e satisfação, Desenvolvimento profissional e benefícios, Ergonomia, Condições de trabalho, e Processo decisório), respondidos por intermédio de uma escala do tipo Likert de cinco pontos. 0 instrumento apresenta estudos de evidências de validade e precisão que atestam suas qualidades psicométricas e, com base no manual do instrumento (Rueda \& Santos, 2011), a partir dos escores obtidos pelos trabalhadores é possível classifica-los em quatro intensidades de percepção de clima organizacional (Baixa, Médio-baixa, Médio-alta, Alta), sendo que quanto maior a pontuação, maior a percepção de um adequado clima de trabalho no contexto organizacional em que atua.

Quanto aos fatores da CLIMOR, o primeiro, Comunicação, integração e satisfação, está associado à qualidade percebida pelos funcionários em termos da eficiente circulação de informações e cooperação entre os trabalhadores e também está relacionado com os sentimentos dos trabalhadores pela organização. No segundo fator, Desenvolvimento profissional e Benefícios, altas pontuações indicam que o funcionário se encontra satisfeito com os aspectos condizentes às políticas de promoção de pessoas e benefícios oferecidos pela organização. 0 terceiro fator, Ergonomia, está associado às preocupações da organização em oferecer a seus funcionários adequadas condições de trabalho, minimizando riscos. Elementos como percepção de adequação em termos salariais e estrutura organizacional são avaliados no quarto fator da CLIMOR, Condições do trabalho. 0 último fator, Processo decisório, 
diz respeito à existência ou não de autonomia na execução das funções laborais percebida pelos trabalhadores (Rueda \& Santos, 2011).

\section{Procedimentos}

Após a aprovação do projeto pelo comitê de ética de pesquisa (CAAE: 45431715.3.0000.5398) os dados foram coletados em organizações de diferentes ramos de atuação, públicas e privadas, no intuito de obter maior variabilidade de respostas. A aplicação dos instrumentos somente ocorreu após os participantes assinarem o Termo de Consentimento Livre e Esclarecido (TCLE) e o tempo médio de aplicação dos instrumentos foi de 15 minutos.

\section{Análise de dados}

Os dados foram analisados por meio de análises descritivas e inferenciais. No que tange às análises descritivas, verificou-se as médias de pontuações entre os instrumentos aplicados, sendo as mesmas classificadas de acordo com as normas contidas nos manuais de tais instrumentos.

Quanto às análises inferenciais verificou-se as diferenças de médias de respostas entre grupos com base no variável tipo de organização (por meio do teste estatístico ANOVA). Também foi utilizada a análise de correlação de Pearson para verificar a associação entre os instrumentos aplicados.

\section{Resultados e Discussão}

A seguir serão apresentados os resultados e discussões, sendo primeiramente elencados os dados obtidos por intermédio da estatística descritiva, com base nos escores da ESUL e CLIMOR. O objetivo foi investigar a classificação média da amostra estudada, para que a partir disso seja possível discutir acerca dos dados. Quanto à ESUL, o instrumento apresenta normas em que se deve levar em consideração o ramo de atuação da organização, ou seja, comércio, indústria e prestação de serviços (Baptista et al., 2016). Os resultados obtidos estão apresentados na Tabela 1.

Tabela 1. Médias dos resultados do teste ESUL

\begin{tabular}{lllll}
\hline Ramo de Atuação & & Média & DP & Classificação \\
\hline \multirow{2}{*}{ Comércio } & Interação & 29,14 & 9,91 & Alta \\
\cline { 2 - 5 } & Benefícios & 7,11 & 5,83 & Média-Baixa \\
\hline \multirow{2}{*}{ Indústria } & Interação & 30,01 & 8,99 & Média-Alta \\
\cline { 2 - 5 } & Benefícios & 12,57 & 4,64 & Média-Baixa \\
\hline \multirow{2}{*}{ Prestação de Serviços } & Interação & 28,58 & 7,92 & Alta \\
\cline { 2 - 5 } & Benefícios & 8,66 & 5,57 & Média-Baixa \\
\hline
\end{tabular}

É possível observar que a média das pontuações no fator Interação do ramo industrial foi classificada como Média-alta, ao passo que em Comércio e Prestação de Serviços as médias obtidas foram consideradas Altas (Baptista et al., 2016). Em relação à importância desse fator no contexto organizacional, Chang (2015), Gillet, Colombat, Michinov, Pronost e Fouquereau (2013) afirmaram que uma característica do suporte laboral é a avaliação subjetiva que um profissional faz em relação 
Pág 71

É sabido (Zagenczyk, Scott, Gibney, Murrell, \& Thatcher, 2010), que a percepção de apoio de ordem afetiva tende a contribuir com maior engajamento por parte dos trabalhadores, bem como com a sensação de bem-estar no contexto laboral. ao tratamento que recebe da organização empregadora, sendo que o componente ligado às interações entre trabalhadores e lideranças se apresenta como um fator altamente relevante na percepção do suporte em sua totalidade.

$\mathrm{Na}$ literatura consultada não foram encontrados estudos que tenham verificado o suporte organizacional em relação ao ramo de atuação das organizações, entretanto é sabido (Zagenczyk, Scott, Gibney, Murrell, \& Thatcher, 2010), que a percepção de apoio de ordem afetiva tende a contribuir com maior engajamento por parte dos trabalhadores, bem como com a sensação de bem-estar no contexto laboral. Uma hipótese acerca das diferenças de classificações encontradas entre os ramos de atuação é de que organizações comerciais e prestadoras, de serviços tendem a promoverem maiores condições para a existência de contatos interpessoais (entre os próprios trabalhadores e também com clientes), ao passo que o ramo industrial, pelas próprias características de produção, tende a promover menos essas possibilidades de relacionamentos interpessoais (tanto entre os próprios trabalhadores, bem como com os clientes).

No fator Benefícios as médias dos três ramos de atuação foram classificadas como Média-baixa (Baptista et al., 2016). Embora alguns autores defendam a ideia de que o suporte de ordem emocional pode mais bem explicar a percepção do suporte laboral como um todo, Alvez, Neiva e Paz (2014) salientam, com base em dados obtidos no estudo conduzido pelas mesmas, que a dimensão instrumental (relacionada a apoios de ordem material) é também um importante preditor do suporte organizacional, podendo essa estar relacionada, dentre outras coisas, com a percepção de bem-estar pessoal, assim como um adequado, ou não, clima organizacional.

Como visto, independentemente no ramo de atuação da organização, os trabalhadores apresentaram uma percepção até certo ponto negativa sobre o segundo fator da ESUL, o que pode ser um indicativo para os gestores no sentido de buscar a implementação de programas de melhorias não somente de remuneração, mas também de benefícios oferecidos pelas organizações (seja no que tange a planos de saúde e odontológicos, bem como condições relacionadas à materiais e equipamentos para a adequada execução das funções de trabalho). Tais ações poderiam favorecer não somente a percepção de bem-estar psicológico para os trabalhadores, mas também físico, podendo com isso evitar adoecimentos e possíveis afastamentos no trabalho (Sá Leitão, Guimarães, \& Rosal, 1998).

Conforme sinalizaram Futris, Schramm, Richardson e Lee (2015), Kurtessis et al. (2017) e Wikhamn e Hall (2014), o suporte organizacional está relacionado à percepção, por parte dos trabalhadores acerca da qualidade do tratamento que recebe da instituição em que exerce suas funções laborais, sendo que questões de ordem emocional (tais como elogios e empatia), bem como de ordem instrumental (como equipamentos adequados para se trabalhar e salários considerados justos para as funções), se bem trabalhadas pela organização, podem ser consideradas importantes preditores de engajamento no trabalho. Em acréscimo, o suporte organizacional também é tido como uma variável que influencia positivamente na percepção da melhoria no relacionamento com a liderança e aumento do desempenho no trabalho (Shanock \& Eisenberger, 2006), à maior percepção de justiça organizacional e confiança entre os funcionários (DeConinck, 2010), com maior percepção de compromisso afetivo e desempenho no trabalho (Casimir, Keith, Wang, \& Ooi, 2014, Kim, Eisenberger, \& Baik, 2016), maior motivação no trabalho (Chen \& Shasser, 2017), maior 
percepção de adequado clima organizacional (Alvez, Neiva, \& Paz, 2014, Balducci \& Kanaane, 2007), dentre outros aspectos.

A avaliação do clima organizacional também foi um dos objetivos da presente pesquisa. Nesse caso, tal temática foi mensurada por intermédio da escala CLIMOR. Embora esse instrumento não tenha como norma de correção a separação dos trabalhadores pelo ramo de atividade da organização (assim como é feito na ESUL), optou-se no presente estudo em se verificar as classificações do clima organização com base na referida variável.

Essa análise da amostra, fazendo-se um recorte com base no ramo de atividade da organização, pode ser considerada um diferencial da pesquisa, uma vez que poucos são artigos publicados na área de Psicologia Organizacional e do Trabalho com esses construtos que realizam análises com base nesse quesito. Conforme destacam Coda (1997) e Zhang, Qiu e Teng (2017), o clima organizacional é um importante variável a ser investigada no contexto laboral quando há o objetivo de se verificar as percepções dos trabalhadores em relação a diferentes aspectos da realidade da organização. Assim, acredita-se que pode haver percepções distintas sobre o clima organizacional e, talvez, o ramo de atividade da organização pode ser uma variável que influencie positiva ou negativamente essa percepção. As informações sobre os resultados da CLIMOR podem ser visualizadas na Tabela 2.

Tabela 2. Médias dos resultados do instrumento CLIMOR

\begin{tabular}{|c|c|c|c|c|}
\hline \multicolumn{2}{|c|}{ Instrumento - CLIMOR } & Média & $D P$ & Classificação \\
\hline \multirow{5}{*}{ Comércio } & $\begin{array}{l}\text { Comunicação, Integração e } \\
\text { Satisfação }\end{array}$ & 38,50 & 10,47 & Média-Baixa \\
\hline & $\begin{array}{l}\text { Desenvolvimento } \\
\text { profissional e Benefícios }\end{array}$ & 22,95 & 9,60 & Média-Baixa \\
\hline & Ergonomia & 20,87 & 7,18 & Média-Baixa \\
\hline & Condições de Trabalho & 11,45 & 3,68 & Média-Baixa \\
\hline & Processo decisório & 9,04 & 3,96 & Média-Baixa \\
\hline \multirow{5}{*}{ Indústria } & $\begin{array}{l}\text { Comunicação, Integração e } \\
\text { Satisfação }\end{array}$ & 37,81 & 9,44 & Média-Baixa \\
\hline & $\begin{array}{l}\text { Desenvolvimento } \\
\text { profissional e Benefícios }\end{array}$ & 26,60 & 8,29 & Média-Baixa \\
\hline & Ergonomia & 22,15 & 5,22 & Média-Baixa \\
\hline & Condições de Trabalho & 11,89 & 4,44 & Média-Baixa \\
\hline & Processo decisório & 9,83 & 3,35 & Média-Baixa \\
\hline \multirow{5}{*}{$\begin{array}{l}\text { Prestação de } \\
\text { Serviços }\end{array}$} & $\begin{array}{l}\text { Comunicação, Integração e } \\
\text { Satisfação }\end{array}$ & 37,95 & 9,33 & Média-Baixa \\
\hline & $\begin{array}{l}\text { Desenvolvimento } \\
\text { profissional e Benefícios }\end{array}$ & 23,90 & 8,84 & Média-Baixa \\
\hline & Ergonomia & 20,74 & 5,84 & Média-Baixa \\
\hline & Condições de Trabalho & 11,17 & 4,02 & Média-Baixa \\
\hline & Processo decisório & 9,94 & 3,61 & Média-Baixa \\
\hline
\end{tabular}


Pág 73

Concorda-se com Robbins (2005) na medida em que 0 autor afirma que a adequada comunicação tende a proporcionar a facilitação no trabalho, levando os indivíduos a perceberem maior qualidade nesse ambiente.
Conforme pode ser visto na Tabela 2, os resultados não indicaram diferenças nas classificações com base no ramo de atividade da organização. Nos três tipos (do comércio, as indústrias e as prestadoras de serviços) os trabalhadores apresentaram classificação Média-Baixa (em todos os fatores da CLIMOR). Villardi, Narducci e Dubeux (2007) salientaram que o clima organizacional influencia diretamente na forma como as pessoas se relacionam no contexto laboral e, nessa mesma direção, uma vez que o construto diz respeito à forma como os trabalhadores percebem a organização, principalmente enquanto um local adequado ou não para execução das funções laborais, os resultados obtidos no presente estudo podem ser considerados preocupantes.

De forma específica, o fator Comunicação, integração e satisfação está associado à qualidade percebida pelos funcionários em relação à circulação de informações e cooperação entre os trabalhadores, assim como aos sentimentos dos funcionários pela organização. 0 resultado encontrado em todos os ramos indica uma percepção não muito adequada desses quesitos na organização (Rueda \& Santos, 2011). Concorda-se com Robbins (2005) na medida em que o autor afirma que a adequada comunicação tende a proporcionar a facilitação no trabalho, levando os indivíduos a perceberem maior qualidade nesse ambiente. Sobre a integração e satisfação, Backes, Lunardi Filho e Lunardi (2006), Bedani (2006) e Abreu, Badanza, Silva e Almeida (2013), ressaltaram que os relacionamentos interpessoais são diretamente intervenientes na percepção do clima organizacional, e que quanto mais acolhedora a organização se apresentar a seus funcionários, maior será a tendência de percepção de um bom local para se trabalhar, aumentando, assim, a satisfação com o contexto laboral.

No segundo fator do instrumento, Desenvolvimento profissional e Benefícios, também houve pontuação Média-Baixa, indicando insatisfação por parte dos trabalhadores quanto aos aspectos condizentes às políticas de promoção de pessoas e benefícios oferecidos pela organização (Rueda \& Santos, 2011). Para Paz (2004) e Paschoal, Torres e Porto (2010), quando não há a percepção de que existam possibilidades de crescimento na organização, também há uma tendência de se diminuir a confiança do trabalhador para com as ações da organização. Sobre os benefícios, Bispo (2006) e Luz (2007) afirmaram que os fatores financeiros são variáveis também responsáveis pela manutenção do adequado clima organizacional, entretanto pode ter uma influência negativa caso os indivíduos não estejam suficientemente contemplados por tais ações.

Pela análise dos resultados no fator Ergonomia foi possível constatar que os trabalhadores ressaltaram que o contexto laboral poderia gerar riscos à saúde, ou seja, não perceberam de forma satisfatória o ambiente físico no trabalho (Rueda \& Santos, 2011). Conforme destacam Canela, Lima e Santiago (2016) e Santos (2016). a ergonomia, que tem por objetivo possibilitar conforto aos indivíduos no trabalho, tende a ser percebida pelo funcionário como um fator positivo em relação ao clima organizacional. Do contrário, havendo más condições ergonômicas, pode acarretar descontentamentos e riscos à saúde física dos trabalhadores.

Elementos como adequação em termos salariais e estrutura organizacional são avaliados no quarto fator da CLIMOR, Condições do trabalho, por meio do qual também foi possível constatar que tais características não foram percebidas de forma adequada (Rueda \& Santos, 2011). Com base em Alves, Neiva e Paz (2014), a satisfação com recursos financeiros oferecidos pela organização é um importante preditor de positivo 
clima organizacional e bem-estar e, em relação às condições de trabalho, Estivalete, Andrade, Faller, Stefanan e Souza (2016) sinalizam que um ambiente seguro para a execução das funções laborais geralmente é associado à percepção positiva de clima organizacional.

O último fator da CLIMOR, Processo decisório, diz respeito à existência ou não de autonomia na execução das funções. A classificação média das respostas foi Média-Baixa, sugerindo que os participantes não percebem elevada autonomia no cotidiano de seus trabalhos (Rueda \& Santos, 2011). Em estudo recente, Chaves e Guimarães (2016) chegaram à conclusão que a promoção da autonomia no trabalho tende a proporcionar, além de um clima agradável no trabalho, também a possibilidade de tornar as funções e decisões nesse contexto mais democráticas.

Também foi objetivo deste estudo realizar análises que pudessem verificar existência de diferenças, estatisticamente significativas, nas médias de respostas entre grupos nos instrumentos aplicados a partir de variáveis sociodemográficas, quais sejam, sexo do participante, idade, tipo de organização (pública, privada e terceiro setor), ramo de atividade da organização (comércio, indústria e prestação de serviços). Dessas variáveis, apenas o tipo organização apresentou diferenças de médias de respostas com significância estatística entre os grupos.

A variável tipo de organização foi composta por três grupos (pública, privada e terceiro setor). Dos dados, as médias de respostas apresentaram diferenças estatisticamente significativas nos fatores Interações (ESUL) e Ergonomia (CLIMOR).

Tabela 3. Distribuição em relação ao tipo de organização

\begin{tabular}{|c|c|c|c|c|c|}
\hline & Instrumento & Tipo de Organização & Média & $\boldsymbol{F}$ & $p$ \\
\hline \multirow{6}{*}{ ESUL } & \multirow{3}{*}{ Interações } & Pública & 26,63 & \multirow{3}{*}{4,298} & \multirow{3}{*}{0,014} \\
\hline & & Privada & 29,69 & & \\
\hline & & Terceiro Setor & 30,55 & & \\
\hline & \multirow{3}{*}{ Benefícios } & Pública & 9,25 & \multirow{3}{*}{0,591} & \multirow{3}{*}{0,554} \\
\hline & & Privada & 9,29 & & \\
\hline & & Terceiro Setor & 7,77 & & \\
\hline \multirow{15}{*}{ CLIMOR } & \multirow{3}{*}{$\begin{array}{l}\text { Comunicação, integração e } \\
\text { satisfação }\end{array}$} & Pública & 36,03 & \multirow{3}{*}{2,46} & \multirow{3}{*}{0,087} \\
\hline & & Privada & 38,82 & & \\
\hline & & Terceiro Setor & 38,41 & & \\
\hline & \multirow{3}{*}{$\begin{array}{l}\text { Desenvolvimento } \\
\text { profissional e Benefícios }\end{array}$} & Pública & 23,31 & \multirow{3}{*}{0,796} & \multirow{3}{*}{0,452} \\
\hline & & Privada & 24,70 & & \\
\hline & & Terceiro Setor & 25,11 & & \\
\hline & \multirow{3}{*}{ Ergonomia } & Pública & 18,97 & \multirow{3}{*}{8,424} & \multirow{3}{*}{0,000} \\
\hline & & Privada & 22,05 & & \\
\hline & & Terceiro Setor & 20,44 & & \\
\hline & \multirow{3}{*}{ Condições de Trabalho } & Pública & 11,06 & \multirow{3}{*}{0,753} & \multirow{3}{*}{0,472} \\
\hline & & Privada & 11,40 & & \\
\hline & & Terceiro Setor & 12,33 & & \\
\hline & \multirow{3}{*}{ Processo decisóro } & Pública & 9,38 & \multirow{3}{*}{0,956} & \multirow{3}{*}{0,386} \\
\hline & & Privada & 9,89 & & \\
\hline & & Terceiro Setor & 10,5 & & \\
\hline
\end{tabular}


Pág 75

Conforme destacam Oliveira et al. (2016), condições inadequadas de trabalho tendem a levar ao adoecimento físico e psíquico dos trabalhadores, seja pela falta de cuidado com a segurança no trabalho, ou mesmo pela escassez de recursos materiais disponíveis aos funcionários.
No fator Interações percebeu-se diferença de médias de respostas entre os grupos com base no variável tipo de organização, sendo que a pontuação mais baixa foi do grupo de trabalhadores das organizações públicas, seguido por privadas e a maior pontuação obtida foi por trabalhadores do terceiro setor. Acerca do suporte laboral, Zagenczyk, Scott, Gibney, Murrell e Thatcher (2010) afirmaram que, por apresentar uma característica subjetiva, sua percepção pode variar de um trabalhador para outro, assim como de um tipo de organização para outra. Em outras palavras, em um mesmo contexto pode haver variações na forma como as pessoas concebem os elementos do suporte.

Destarte, não é possível afirmar que o tipo de organização é uma variável de grande responsabilidade pela maior ou menor percepção de suporte, neste caso, no que tange às interações humanas no trabalho. Os estudos recentes que fizeram avaliações da percepção de suporte em relação ao tipo de organização não encontraram um resultado comum. Em acréscimo, no estudo de Formiga, Fleury e Souza (2014) a percepção de suporte quanto às interações humanas no trabalho foi superior em trabalhadores de empresas privadas, ao passo que Formiga et al. (2015) encontraram maior percepção de suporte na referida dimensão em trabalhadores de organizações públicas e, por fim, Oliveira, Lins, Silva e Fontoura (2016) não encontrou diferenças significativas em relação aos tipos de organizações avaliadas.

Na dimensão Ergonomia, da CLIMOR, também houve diferenças nas médias de respostas em relação aos grupos analisados, sendo que trabalhadores de instituições públicas novamente obtiveram a menor média, seguido por terceiro setor e indivíduos de organizações privadas. Esse dado indica, segundo Rueda e Santos (2011), que trabalhadores de instituições públicas perceberam que suas organizações se preocupam menos com questões ligadas ao conforto e segurança de seus funcionários, quando comparados com trabalhadores provenientes de outros tipos de organizações. Na literatura consultada não foram encontrados estudos que fizeram análises sobre o clima organizacional, em especial na variável ergonomia, levando-se em conta às instituições de trabalho, entretanto, conforme destacam Oliveira et al. (2016), condições inadequadas de trabalho tendem a levar ao adoecimento físico e psíquico dos trabalhadores, seja pela falta de cuidado com a segurança no trabalho, ou mesmo pela escassez de recursos materiais disponíveis aos funcionários.

A seguir, a Tabela 4 apresentará as análises de correlações entre os instrumentos aplicados. De forma geral, pode-se perceber que houve somente correlações positivas entre os instrumentos de clima organizacional e suporte laboral. Isso significa que, quanto maior a pontuação no instrumento de clima organizacional, maior tende a ser a pontuação no instrumento de suporte, e vice-versa (Baptista, Rueda, Bartholomeu, Pires, \& Rochael, 2010). Das 28 associações entre as escalas, nove foram de magnitude fraca (entre 0 e 0,39), 14 de magnitude moderada (entre 0,40 e 0,69) e cinco de magnitude forte (superiores a 0,70), com base na classificação de correlação proposta por Dancey e Reidy (2006).

Associações positivas entre suporte organizacional e clima organizacional já foram documentadas na literatura científica em outros estudos. A exemplo, Sousa, Mendonça, Zanini e Nazareno (2009) ao encontrarem correlações positivas entre os dois construtos, salientaram que quando o suporte organizacional é percebido pelos trabalhadores de forma positiva, os mesmos passam a se sentir valorizados e tendem a desempenhar melhor suas funções. Nessa mesma direção, alguns autores (Dessen, \& Paz, 2010, Hayton, Carnabuci \& Eisenberger, 2012, Liu, Hu, Wang, Sui, \& 
Ma, 2013, Sant'anna, Paschoal, \& Gosendo, 2012, Wang, Tung, \& Hsieh, 2013, Zhang et al., 2017) destacaram que as condições mais favoráveis para o desenvolvimento de adequada percepção de suporte organizacional estão relacionadas ao quanto os trabalhadores percebem que a organização se preocupa, cuida de seu bem-estar e o valoriza.

Tabela 4. Correlações entre os instrumentos ESUL e CLIMOR

\begin{tabular}{llll}
\hline \multicolumn{1}{c}{ Interações } & \multicolumn{2}{c}{$\begin{array}{c}\text { ESUL } \\
\text { Benefícios }\end{array}$} \\
\hline \multirow{3}{*}{ CLIMOR } & Comunicação, integração e satisfação & $0,72^{* *}$ & $0,24^{* *}$ \\
\cline { 2 - 4 } & Desenvolvimento profissional e Benefícios & $0,58^{* *}$ & $0,59^{* *}$ \\
\cline { 2 - 4 } & Ergonomia & $0,55^{* *}$ & $0,32^{* *}$ \\
\cline { 2 - 4 } & Condições de Trabalho & $0,22^{* *}$ & $0,34^{* *}$ \\
\cline { 2 - 4 } & Processo Decisório & $0,54^{* *}$ & $0,24^{* *}$ \\
\hline${ }_{*}^{*} p<0,001$. & & & \\
&
\end{tabular}

De toda forma, tendo como base as análises de correlação do presente estudo, bem como a literatura científica utilizada para a discussão, para fins práticos, ressalta-se acerca da importância das organizações, principalmente por intermédio dos departamentos de Gestão de Pessoas, refletirem e implementarem ações que possam fazer com que os trabalhadores percebam cada vez mais o suporte oferecido pela organização, uma vez que essas tendem a proporcionar um clima organizacional mais adequado. Em complemento a essas sugestões, Paschoal, Torres e Porto (2010) afirmaram que o suporte organizacional é um importante variável a ser analisada no processo de compreensão sobre a forma como os trabalhadores percebem o ambiente de trabalho, ou seja, elementos do clima organizacional. De forma adicional, variáveis como relacionamento entre funcionários, salários, benefícios, condições de trabalho e relacionamento com a liderança, se bem abordados, estarão associados a maiores indicadores de suporte laboral (Estivalete et al., 2016, Silva, Cappellozza, \& Costa, 2014, Silva \& Ferreira, 2013).

Em estudo realizado Kolb (2014) é destacado que o clima organizacional é influenciado por diversos fatores, e que o suporte laboral é uma variável que o influencia positivamente. De forma semelhante, outros autores também constataram correlações positivas entre suporte laboral e clima organizacional (Hashish, 2015, Köse, 2016. Putter, 2010, Rosès, 2013). Obviamente que não é possível inferir com grande margem de confiança, por meio da análise de correlação, uma relação causal e explicativa dos construtos investigados. Dessa forma, uma sugestão para estudos futuros seja a realização de análises de regressão, a fim de verificar o poder explicativo de um construto em relação ao outro.

\section{Considerações Finais}

A presente pesquisa se propôs a analisar a relação entre as temáticas clima organizacional e suporte laboral, por meio da aplicação de dois instrumentos em uma amostra de trabalhadores. Ao longo do estudo pode-se verificar que os objetivos foram alcançados, obtendo-se dados que corroboraram com achados da literatura e permitiram inferir acerca de uma melhor reflexão dos construtos. Tanto o suporte organizacional como o clima organizacional podem ser considerados variáveis protetivas, quando percebidas de forma positiva pelos trabalhadores. Assim, uma organização de trabalho que busca a valorização e reconhecimento de seus funcionários, seja 
por meio de remuneração, condições de trabalho e benefícios (suporte material) ou mesmo pelo aspecto interacional (suporte afetivo), tende a proporcionar bem-estar a seu quadro de trabalhadores, o que pode ser visto como um diferencial de mercado. Na mesma direção, o funcionário que percebe apoio por parte de sua organização provavelmente buscará atuar no sentido de continuidade desse ambiente laboral agradável, o que tende a melhorar não somente a produtividade organizacional, mas também a sensação de qualidade de vida no trabalho.

Tendo em vista os resultados da pesquisa, recomenda-se a realização de estudos futuros com o intuito de aprofundar os resultados encontrados, utilizando-se de amostras maiores a fim de comparar os dados obtidos, principalmente no que tange à separação amostral pelo ramo de atividade da organização. Embora o presente estudo tenha tido como diferencial a análise da amostra de acordo com o ramo de atividade da organização, investigações com um público maior poderão proporcionar inferências mais consistentes. Por fim, sugere-se que sejam realizados estudos longitudinais visando verificar possíveis influências dos construtos suporte laboral e clima organizacional em indicadores de absenteísmo, rotatividade e saúde mental dos trabalhadores.

\section{Referências}

Abreu, N. R., Baldanza, R. F., Silva, E. S., \& Almeida, N. H. S. (2013). Análise dos fatores intraorganizacionais influenciadores no clima organizacional em um hospital universitário. NACE, 12(1), 39-70. Disponível em: https://dialnet.unirioja.es/servlet/articulo?codigo $=5160831$.

Alves, V. D., Neiva, E. R., \& Paz, M. G. T. (2014). Configurações de Poder, Suporte Organizacional e Bem-Estar Pessoal em uma Organização Pública. Psicologia em Pesquisa, 8(2), 159-169. doi: https://doi.org/10.5327/Z1982-1247201400020005

Amazue, L. O., \& Onyishi, I. E. (2016). Stress coping strategies, perceived organizational support and marital status as predictors of work-life balance among nigerian bank employees. Social Indicators Research, 128(1), 147-159. doi: https:// doi.org/10.1007/s11205-015-1023-5

Aselage, J., \& Eisenberger, R. (2003). Perceived organizational support and psychological contracts: A theoretical integration. Journal of Organizational Behavior, 24(5), 491 - 509. doi: https://doi.org/10.1002/job.211

Azevedo, M. C., \& Tonell, M. J. (2014). Os diferentes contratos de trabalho entre trabalhadores qualificados brasileiros. Revista de Administração Mackenzie - RAM, 15(3), 191-220. doi: http://dx.doi.org/10.1590/1678-69712014/administracao. v15n3p191-220

Backes, D. S., Lunardi Filho, W. D., \& Lunardi, V. L. (2006). O processo de humanização do ambiente hospitalar centrado no trabalhador. Revista da Escola de Enfermagem USP, 40(2), 221-227. doi: http://dx.doi.org/10.1590/S0080-62342006000200010

Balducci, D., \& Kanaane, R. (2007). Relevância da gestão de pessoas no clima organizacional de uma empresa de engenharia. Boletim da Academia Paulista de Psicologia, XXVII(2), 133-147. Disponível em: http://pepsic.bvsalud.org/scielo. php?script=sci arttext\&pid=S1415-711X2007000200012.

Baptista, M. N. Cardoso, H. F., Santos, A. A. A., \& Noronha, A. P. P. (2016). ESUL: Escala de Suporte Laboral. Editora Hogrefe. São Paulo.

Baptista, M. N., Rueda, F. J. M., Bartholomeu, D., Pires, S. D., \& Rochael, F. (2010) Suporte laboral e identificação organizacional: um estudo de validade. Aletheia, 32, 53-69. Disponível em: http://pepsic.bvsalud.org/scielo.php?script=sci arttext\&pid=S1413-03942010000200005. 
Bispo, C. A. F. (2006). Um novo modelo de pesquisa de clima organizacional. Produção, 16(2), 258-273. doi: https://doi.org/10.1590/S0103-65132006000200007

Bowling, A. (1997). Measuring health: a review of quality of life measurement scales. Philadelphia: Open University.

Bedani, M. (2006). Clima Organizacional: investigação e diagnóstico: estudo de caso em agência de viagens e turismo. Revista Psicología para América Latina, 7, 49-56. Disponível em: http://pepsic.bvsalud.org/scielo.php?script=sci_arttext\&pid $=$ S1870-350X2006000300011.

Canela, D. C. S., Lima, M. C. R., \& Santiago, A. M. S. (2016). Clima organizacional: estudo de caso em instituto federal de educação: ciência e tecnologia. Id on Line Revista Multidisciplinar e de Psicologia, 10(30), 210-224. doi: https://doi. org/10.14295/idonline.v10i30.436

Casimir, G., Keith, Y. N., Wang, K. Y., \& Ooi, G., (2014). The relationships amongst leader-member exchange, perceived organizational support, affective commitment, and in-role performance: A social-exchange perspective, Leadership \& Organization Development Journal, 35(5), 366-385. doi: https://doi.org/10.1108/LODJ-04-2012-0054

Chang, C. (2015). Moderating Effects of Nurses' Organizational Support on the Relationship Between Job Satisfaction and Organizational Commitment. Western Journal of Nursing Research, 37(6), 724-745. doi: https:// doi.org/10.1177/0193945914530047

Chaves, J. A., \& Guimarães, M. G. V. (2016). Análise do clima organizacional em centro cirúrgico de um Hospital Universitário da cidade de Manaus. Revista FAROL, 1(1), 212-219. Disponível em: https://revistas.face.ufmg. br/index.php/farol/index.

Chen, Y., \& Shasser, M. A. (2017). The influences of perceived organizational support and motivation on self-initiated expatriates' organizational and community embeddedness. Journal of World Business, 52(2), 197-208. doi: https://doi.org/10.1016/j.jwb.2016.12.001

Chermack, T. J., Coons, L. M., Nimon, K., Bradley, P., \& Glick, M. B. (2015). The Effects of Scenario Planning on Participant Perceptions of Creative Organizational Climate. Journal of Leadership \& Organizational Studies, 22(3), 355-371. doi: https://doi.org/10.1177/1548051815582225

Coda, R. (1997). Pesquisa de clima organizacional e gestão estratégica de recursos humanos. In: Bergamini, C. W., \& Coda, R. (org.). Psicodinâmica da vida organizacional: motivação e liderança. São Paulo: Atlas.

Culié, J., Khapova, S. N., \& Arthur, M. B. (2014). Careers, clusters and employment mobility: The influences of psychological mobility and organizational support. Journal of Vocational Behavior, 84(2), 164-176. doi: http://dx.doi.org/10.1016/j. jvb.2014.01.002

Dancey, C. P., \& Reidy, J. Estatística sem Matemática para Psicologia: usando SPSS para Windows. 3 a ed. Porto Alegre: Artmed, 2006.

DeConinck, J. B. (2010). The effect of organizational justice, perceived organizational support, and perceived supervisor support on marketing employees' level of trust. Journal of Business Research, 63(12), 1349-1355. doi: https://doi.org/10.1016/j.jbusres.2010.01.003 
Dessen, M. C., \& Paz, M. G. T. (2010). Validação do instrumento de indicadores de bem-estar pessoal nas organizações. Psicologia em Estudo, 15, 409-418. Disponível em: http://www.scielo.br/pdf/pe/v15n2/a20v15n2.

Eisenberger, R., Fasolo, P., \& Davis-LaMastro, V. (1990). Perceived organizational support and employee diligence, commitment, and innovation. Journal of Applied Psychology, 75(1), 51-59. Disponível em: http://www. drillscience.com/DPS/OrganizationalSupportAndDiligence.pdf.

Eisenberger, R., Huntington, R., Hutchison, S., \& Sowa, D. (1986). Perceived organizational support. Journal of Applied Psychology, 71, 500-507. Disponível em: http://classweb.uh.edu/eisenberger/wp-content/uploads/ sites/21/2015/04/22 Perceived Organizational Support.pdf.

Eisenberg, R., Stinglhamber, F., Vandenberghe, C., Sucharski, I. L., \& Rhoades, L. (2002). Perceived supervisor support: Contributions to perceived organizational support and employee retention. Journal of Applied Psychology, 87(3), 565-573. doi: https://doi.org/10.1037//0021-9010.87.3.565

Estivalete, V. F. B., Andrade, T., Faller, L. P., Stefanan, A. A., \& Souza, D. L. (2016). Suporte social e suporte organizacional como antecedentes do bem-estar no trabalho: a perspectiva de colaboradores de uma empresa de logística ferroviária. Revista de Administração da UNIMEP, 14(2), 31-56. doi: https:// doi.org/10.15600/1679-5350/rau.v14n2p31-56

Formiga, N. S., Bassanin, L. L., Souza, M. V., Silva, F. M. C., Silva, G. G. C., \& Souza, M. A. (2015). Suporte organizacional em trabalhadores de diferentes empresas: comprovação de uma medida. Revista de Psicología Universidad de Antioquia, 7(1), 9-20. Disponível em: http://aprendeenlinea.udea. edu.co/revistas/index.php/psicologia/article/view/25260/20870.

Formiga, N., Fleury, L. F. O., \& Souza, M. A. (2014). Evidências de validade da escala de percepção de suporte organizacional em funcionários de empresa pública e privada. Estudos Interdisciplinares em Psicologia, 5(2), 60-76. doi: https://doi.org/10.5433/2236-6407.2014v5n2p34.

Futris, T. G., Schramm, D. G., Richardson, E. W., \& Lee, T. K. (2015). The impact of organizational support on the transfer of learning to practice. Children and Youth Services Review, 51, 36-43. doi: http://dx.doi.org/10.1016/j. childyouth.2015.01.019.

Gillet, N., Colombat, P., Michinov, E., Pronost, A., \& Fouquereau, E. (2013). Procedural justice, supervisor autonomy support, work satisfaction, organizational identification and job performance: the mediating role of need satisfaction and perceived organizational support. Journal of Advanced Nursing, 69(11), 2560-2571. doi: https://doi.org/10.1111/jan.12144.

Gillet, N., Huart, I., Colombat, P., \& Fouquereau, E. (2013). Perceived organizational support, motivation, and engagement among police officers. Professional Psychology: Research and Practice, 44(1), 46-55. doi: http://dx.doi. org/10.1037/a0030066.

Gomide Jr, S., Guimarães, L., \& Damásio, L. (2004). Construção e validação de um instrumento de medida de percepção de suporte social no trabalho. II Seminário GIBEST.

Hashish, E. A. A. (2015). Relationship between ethical work climate and nurses' perception of organizational support, commitment, job satisfaction and turnover intente. Nursing Ethics, 24(2), 151-166. doi: $10.1177 / 0969733015594667$. 
Hayton, J. C., Carnabuci, G., \& Eisenberger, R. (2012). With a little help from my colleagues: a social embeddedness approach to perceived organizational support. Journal of Organizational Behavior, 33, 235-249. doi: https://doi.org/10.1002/ job.755.

Kim, K. Y., Eisenberger, R., \& Baik, K. (2016). Perceived organizational support and affective organizational commitment: Moderating influence of perceived organizational competence. Journal of Organizational Behavior, 37(4), 558-583. doi: https://doi.org/10.1002/job.2081.

Kolb, D. A. (2014). Experiential learning: Experience as the source of learning and development. Pearson Education.

Köse, A. (2016). The Relationship between Work Engagement Behavior and Perceived Organizational Support and Organizational Climate. Journal of Education and Practice, 7(27), 42-52. Disponível em: https://files.eric.ed.gov/fulltext/ EJ1115916.pdf.

Kurtessis, J. N., Eisenberger, R., Ford, M. T., Buffardi, L. C., Stewart, K. A., \& Adis, C. S. (2017). Perceived Organizational Support: A Meta-Analytic Evaluation of Organizational Support Theory. Journal of Management, 43(6), 1854-1884. doi: https:// doi.org/10.1177/0149206315575554

Liu, L., Hu, S., Wang, L., Sui, G., \& Ma, L. (2013). Positive resources for combating depressive symptoms among Chinese male correctional officers: perceived organizational support and psychological capital. BMC Psychiatry, 13, 1-9. doi: https:// doi.org/10.1186/1471-244X-13-89

Luz, R. (2007). Gestão do clima organizacional. 3. ed. Rio de Janeiro: Quality.

Menezes, I. M., \& Gomes, C. P. (2010). Clima Organizacional: uma revisão histórica do construto. Psicologia em Revista, 16(1), 158-179. doi: https://doi.org/10.5752/ P.1678-9563.2010v16n1p158

Nakata, L. E., Veloso, E. F. R, Fischer, A. L., \& Dutra, J. S. (2009). Uso de pesquisas de clima organizacional no Brasil. Gestão Contemporânea, 6(6), 45-68. Disponível em: https://bdpi.usp.br/single.php? id=001823542.

Oliveira, T. F., Lins, V. L., Silva, R. M., \& Fontoura, L. V. (2016). Qualidade de vida no trabalho: um estudo comparativo entre professores de escola pública e privada. Psicologia Argumento, 34(85), 104-119. doi: https://doi.org/10.7213/psicol.argum.34.085.A002

Paschoal, T., Torres, C. V., \& Porto, J. B. (2010). Felicidade no trabalho: relações com suporte organizacional e suporte social. Revista de Administração Contemporânea, 14(6), 1054-1072. doi: https://doi.org/10.1590/S1415-65552010000700005

Paz, M. G. T. (2004). Poder e saúde organizacional. In A. Tamayo (Org.). Cultura e saúde nas organizações. Porto Alegre: Artmed.

Putter, L. (2010). The relation between organizational climate and performance and an investigation of the antecedents of organizational climate. (Master's Thesis). Delft Technology University, Delft.

Ribeiro, J. L. P. (1999). Escala de satisfação com o suporte social. Análise psicológica, 3(17), 547-558. Disponível em: http://www.scielo.mec.pt/pdf/aps/ v17n3/v17n3a10.pdf.

Robbins, S. P. (2005). Comportamento organizacional. $11^{\text {a }}$ ed. São Paulo: Pearson Prentice Hall.

Rosès, C. F. (2013). Estudos para a construção de uma escala de avaliação de clima no ambiente de trabalho - ECAT. Tese de doutorado. Universidade São Francisco. Itatiba.

Rueda F. J. M., \& Santos, A. A. A. (2011). Escala de Avaliação do Clima Organizacional (CLIMOR). Vetor Editora, São Paulo. 
Rueda, F. J. M., Santos, A. A. A., Raad, A. J., Ávila-Batista, A. C., \& Nery, J. C. S. (2014). Relación entre los constructos clima organizacional, apoyo laboral y salud organizacional en una muestra de estudiantes que trabajan. Ciencias Psicológicas, 8(1), 7-16. Disponível em: https://www.redalyc.org/articulo.oa?id=459545412002.

Sá Leitão, J., Guimarães, T., \& Rosal, M. A. (1998). Metodologia de diagnóstico de clima organizacional em ambiente de inovação tecnológica. Associação Nacional dos Programas de Pós-Graduação em Administração (Org.), XXII Encontro Anual da Associação Nacional dos Programas de Pós-Graduação em Administração - ENANPAD. Disponível em: http://www.anpad.org.br/admin/pdf/enanpad1998-rh-17.pdf.

Sant'anna, L. L., Paschoal, T., \& Gosendo, E. E. M. (2012). Bem-estar no trabalho: relações com estilos de liderança e suporte para ascensão, promoção e salários. Revista de Administração Contemporânea, 16(5), 744-764. doi: https://doi.org/10.1590/ S1415-65552012000500007

Santos, V. B. (2016). A percepção do clima organizacional dos servidores públicos, sob a ótica da ergonomia: um estudo de caso nas recém-criadas coordenações de infraestrutura, financças e compras da UFPE. Dissertação de mestrado. Universidade Federal de Pernambuco. Recife.

Sears, G. J., Zhang, H., \& Han, Y. (2016). Negative affectivity as a moderator of perceived organizational support - work outcome relationships. Personality and Individual Differences, 98, 257-260. doi: https://doi.org/10.1016/j.paid.2016.04.026

Serviço Brasileiro de Apoio às Micro e Pequenas Empresas - SEBRAE. (2013). Anuário do trabalho na micro e pequena empresa, 6. ed. Brasília, DF: DIEESE. Disponível em: http://www.sebrae.com.br/Sebrae/Portal\%20Sebrae/Anexos/Anuario\%20 do\%20Trabalho\%20Na\%20Micro\%20e\%20Pequena\%20Empresa 2013.pdf.

Shanker, R., Bhanugopan, R., Heijden, B. I. J. M., \& Farrell, M. (2017). Organizational climate for innovation and organizational performance: The mediating effect of innovative work behavior. Journal of Vocational Behavior, 100, 67-77. doi: https:// doi.org/10.1016/j.jvb.2017.02.004

Shanock, L. R., \& Eisenberger, R. (2006). When supervisors feel supported: Relationships with subordinates' perceived supervisor support, perceived organizational support, and performance. Journal of Applied Psychology, 91(3), 689-695. doi: https://doi. org/10.1037/0021-9010.91.3.689

Shmidt, M. S. F., \& Frazier, L. (2016). What Facilitates Dynamic Capabilities? The Role of Organizational Climate for Trust. Long Range Planning, 50(5), 550-566. doi: https://doi.org/10.1016/j.Irp.2016.05.005

Shoss, M. K., Eisenberger, R., Restubog, S. L. D., \& Zagenczyk, T. J. (2013). Blaming the organization for abusive supervision: The roles of perceived organizational support and supervisor's organizational embodiment. Journal of Applied Psychology, 98(1), 158-168. doi: http://dx.doi.org/10.1037/a0030687

Simões, P. H. C., Alves, J. E. D., \& Silva, P. L. N. (2016). Transformações e tendências do mercado de trabalho no Brasil entre 2001 e 2015: paradoxo do baixo desemprego?. Revista Brasileira de Estudos de População, 33(3), 541-566. doi: http:// dx.doi.org/10.20947/s0102-30982016c0005

Silva, C. A., \& Ferreira, M. C. (2013). Dimensões e indicadores da qualidade de vida e do bem-estar no trabalho. Psicologia: Teoria e Pesquisa, 29(3), 331-339. Disponível em: http://www.scielo.br/pdf/ptp/v29n3/v29n3a11.pdf.

Silva, R. S., Cappellozza, A., \& Costa, L. V. (2014). 0 Impacto do Suporte Organizacional e do Comprometimento Afetivo Sobre a Rotatividade. Revista de Administração IMED, 4(3), 314-329. Disponível em: https://dialnet.unirioja.es/servlet/articulo? codigo $=5061334$. 
Sousa, I. F., Mendonça, H., Zanini, D. S., \& Nazareno, E. (2009). Estresse ocupacional, Coping e Burnout. Estudos, Vida e Saúde, 36(1/2), 57-74. doi: https://doi. org/10.18224/est.v36i1.1018

Viitala, R., Tanskanen, J., \& Säntti, R. (2015) The connection between organizational climate and well-being at work. International Journal of Organizational Analysis, 23(4), 606-620, doi: https://doi.org/10.1108/IJOA-10-2013-0716

Villardi, B. Q., Narducci, V., \& Dubeux, V. J. C. (2007). O desenvolvimento qualitativo e quantitativo de uma ferramenta para o diagnóstico de clima organizacional que subsidie Programas Motivacionais no Poder Judiciário: Os Traços Culturais de um Tribunal Regional. Encontro da ANPAD, 31. Disponível em: http://www.anpad. org.br/admin/pdf/APS-A1021.pdf.

Wallace, J. C., Edwards, B. D., Paul, J., Burke, M., Christian, M., \& Eissa, G. (2016). Change the Referent? A Meta-Analytic Investigation of Direct and Referent-Shift Consensus Models for Organizational Climate. Journal of Management, 42(4), 838-861. doi: https://doi.org/10.1177/0149206313484520.

Wang, Y., Tung, C., Hsieh, H. (2013). Organizational ethical climate, perceived organizational support, and employee silence: A cross-level investigation. Human relations 66(6) 783-802. doi: https://doi.org/10.1177/0018726712460706.

Wayne, S., Shore, L. M., Bommer, W. H., \& Tetrick, L. E. (2002). The role of fair treatment and rewards in perceptions of organizational support and leader-member exchange. Journal of applied psychology, 87(3), 590- 598. doi: https://doi. org/10.1037//0021-9010.87.3.590.

Wikhamn, W., \& Hall, A. T. (2014). Accountability and satisfaction: organizational support as a moderator. Journal of Managerial Psychology, 29(5), 458-471. doi: https://doi.org/10.1108/JMP-07-2011-0022.

Zagenczyk, T. J., Scott, K. D., Gibney, R., Murrell, A. J., \& Thatcher, J. B. (2010). Social influence and perceived organizational support: a social networks analysis. Organizational Behavior and Human Decision Processes, 111(2), 127-138. doi: https:// doi.org/10.1016/j.obhdp.2009.11.004.

Zanelli, J. C., \& Bastos, A. V. B. (2004). Inserção profissional do psicólogo em organizações e no trabalho. In: Zanelli, J. C., Borges-Andrade, J. E., \& Bastos, A. V. B. (Orgs.) Psicologia, Organizações e Trabalho. Porto Alegre: Artes Médicas.

Zhang, L., Qiu, Y., \& Teng, E. (2017). Cross-level relationships between justice climate and organizational citizenship behavior: perceived organizational support as mediator. Social Behavior and Personality, 45(3), 387-398. doi: https://doi. org/10.2224/sbp.4842. 